\title{
TOXICIDADE DETERMINADA PELO USO DOS AGROTÓXICOS \\ EM ORGANISMOS INDICADORES DE QUALIDADE DA ÁGUA
}

TOXICITY DETERMINED BY THE USE OF AGROCHEMICALS IN ORGANISMS INDICATORS OF WATER QUALITY

\author{
Luciana de Castro \\ Nascimento (i) \\ Engenheira Ambiental, \\ Universidade Federal do \\ Tocantins (UFT) - Palmas (TO), \\ Brasil.
}

\section{Liliana Pena Naval (D)}

Professora coordenadora do Laboratório de Saneamento Ambiental, Universidade Federal do Tocantins (UFT) - Palmas (TO), Brasil.

\section{Endereço para correspondência:}

Liliana Pena Naval - Laboratório de Saneamento Ambiental, Universidade Federal do Tocantins Avenida NS 15, 109 Norte Bloco 2, Sala 7 - CEP 77001-090 Palmas (TO), Brasil E-mail: liliana@mail.uft.edu.br

Recebido em: 24/04/2019

Aceito em: 11/10/2019

\section{RESUMO}

A expansão das fronteiras agrícolas tem promovido o aumento de áreas cultivadas e uso de agrotóxicos, e inquietações quanto ao destino destes tem sido manifestadas, sobretudo pela exposição a que são submetidos os corpos hídricos. Admitindo-se a preocupação, objetivou-se avaliar o grau de toxicidade, determinado pelo uso dos agrotóxicos glifosato, atrazina e imidacloprido, em organismos indicadores de qualidade da água. Para isso, realizaram-se testes de sensibilidade e definitivos empregando-se dafinídeos (Daphnia magna). Os testes de sensibilidade apresentaram concentração efetiva $\left(\mathrm{CE}_{50}\right)$ de $0,77 \mathrm{mg} \cdot \mathrm{L}^{-1}$, atestando sua utilização e o preconizado pela ISO 6341. Para os testes definitivos e concentrações testadas, foi encontrada $\mathrm{CE}_{50}$ para o glifosato de $27,4 \mathrm{mg} \cdot \mathrm{L}^{-1}$, para a atrazina $\mathrm{CE}_{50}$ de $8,1 \mathrm{mg} \cdot \mathrm{L}^{-1}$ e para o imidacloprido $217,1 \mathrm{mg} \cdot \mathrm{L}^{-1}$. Como efeito deletério, observou-se que, após os testes, os organismos imóveis apresentaram deformações nas carapaças e no tubo digestivo. Entre as propriedades mais agressivas dos agrotóxicos, destacou-se sua miscibilidade.

Palavras-chave: herbicida; inseticida; bioindicadores; toxicologia.

\section{ABSTRACT}

The expansion of agricultural frontiers has promoted the increase of cultivated areas and the use of agrochemicals; concerns about their destination have been manifested, mainly by the exposure to which water bodies are submitted. Admitting the concern, the objective was to evaluate the degree of toxicity, determined by the use of pesticides glyphosate, atrazine and imidacloprid, in organisms that indicate water quality. For this, sensitivity and d/efinitive tests were carried out using dafinids (Daphnia magna). Sensitivity tests showed an EC50 of $0.77 \mathrm{mg} \cdot \mathrm{L}^{-1}$, attesting to its use and that recommended by ISO 6341. For the final tests and concentrations tested, an EC50 was found for glyphosate of $27.4 \mathrm{mg} \cdot \mathrm{L}^{-1}$, for atrazine an EC50 of $8.1 \mathrm{mg} \cdot \mathrm{L}^{-1}$ and $217.1 \mathrm{mg} \cdot \mathrm{L}^{-1}$ for imidacloprid. As a deleterious effect, it was observed that after the tests, the immobile organisms presented deformations in the carapaces and in the digestive tract. Among the properties of the most aggressive pesticides, their miscibility was highlighted.

Keywords: herbicide; insecticide; environmental bioindicator; toxicology. 


\section{INTRODUÇÃO}

Ao longo das últimas décadas, o aumento de áreas cultivadas e do uso de agrotóxicos vem se tornando motivo de inquietação e preocupação quanto ao destino destes no ambiente (RIBEIRO et al., 2007). Os mananciais, tanto superficiais quanto subterrâneos, estão expostos aos agrotóxicos aplicados. Quando se trata de produção agrícola, o Brasil ganha lugar de destaque, tendo em vista que o próprio modelo de agricultura adotado induz a um desenvolvimento voltado para ganhos de produtividade. Os impactos gerados afetam tanto o meio ambiente quanto a saúde humana, os quais têm sido desprezados, bem como a contaminação dos recursos ambientais (FERREIRA, 2014).

O ambiente, ao receber material biológico ou substâncias químicas por processos de despejo e/ou lixiviação, poderá sofrer ações de degradação, em alguns casos, de forma irreversível (KHATRI; TYAGI, 2015). Muitos desses processos podem levar à morte da biota aquática, quebras nas cadeias tróficas, a eutrofização dos corpos hídricos e ainda inviabilizar ou comprometer os diversos usos da água, além de ocasionar desequilíbrios ecológicos e biológicos, como, por exemplo, a contaminação de aquíferos e lençóis freáticos. Caso a contaminação se dê por agrotóxicos, considerar o grau de persistência dos contaminantes é relevante. Em geral, os pesticidas podem persistir no ambiente por longos períodos, podendo ser bioacumuláveis, tóxicos e persistentes.

A presença de glifosato em águas subterrâneas foi verificada, principalmente em regiões produtoras de soja (MERCURIO et al., 2014). O glifosato (N- (fosfonometil) glicina) é um herbicida de amplo espectro, sendo o mais utilizado (OSTEN; DZUL-CAAMAL, 2017). Cultivares geneticamente modificados, com resistência ao glifosato, como é o caso da soja, levam ao uso indiscriminado desse agrotóxico, porque a dependência transgênica de sementes-glifosato não pode ser separada. O glifosato apresenta como principais metabólitos o ácido aminometilfosfônico (AMPA) e o glioxilato.

A presença do AMPA na atmosfera (BATTAGLIN et al., 2014), em águas superficiais (COUPE et al., 2012), sedimentos (RONCO et al., 2016) e águas subterrâneas rasas (VAN STEMPVOORT et al., 2016) tem sido observada.

A exposição ao agrotóxico pode ter efeitos como irritação da pele, falta de ar, espasmos musculares e pro- blemas genéticos (USEPA, 2017). Estudos indicam uma correlação entre o glifosato e os riscos genotóxicos, hormonais, enzimáticos (DARUICH et al., 2001; RICHARD et al., 2005), reprodutivos (MARC et al., 2002) e ainda apresenta associação positiva com a indução de tumores e, por isso, foram classificados como provavelmente carcinogênicos para seres humanos (IARC, 2015).

Outros agrotóxicos cujo uso é comum são a atrazina e o imidacloprido. A atrazina possui moderada mobilidade e elevada persistência no solo (os valores variam entre 100 a $124 \mathrm{Koc}$ ) (DORES; DE-LAMONICA-FREIRE, 2001), assim como o imidacloprido, também caracterizado com mobilidade moderada, entre 132 a $310 \mathrm{Koc}$ (CDPR, 2016). Esses fatores contribuem para a acumulação a níveis contaminantes, tanto na superfície quanto em profundidade nos solos, potencialmente atingindo águas superficiais e subterrâneas (LIU et al., 2016; PROSEN, 2012).

Destaca-se que o imidacloprido apresenta alta variabilidade no solo (KURWADKAR et al., 2013), a meia-vida está entre 28 e 1.250 dias, e a perda em áreas agrícolas, em geral, dá-se por meio da degradação ou lixiviação nas águas (GOULSON, 2013). Imediatamente após a aplicação, pode ocorrer a lixiviação dos neonicotinóides (classe de inseticidas a que pertence o imadacloprido), de modo que níveis significativos podem ser previstos nas águas subterrâneas, particularmente se houver chuvas fortes nesse momento (THUYET et al., 2012).

Essas alterações podem ser verificadas por meio da determinação da concentração dos diferentes compostos presentes no ambiente, que normalmente se dá por análises físicas, químicas e microbiológicas, mas se pode ainda empregar bioindicadores (PARMAR et al., 2016; TSYGANKOV et al., 2017) na busca de repostas mais precisas e rápidas, os quais se mostram eficazes e são usados cada vez mais frequentemente (HOLT; MILLER, 2010; ARRAES; LONGHIN, 2012). A indicação dos fatores ambientais bióticos ou abióticos por meio de sistemas biológicos é o que se pode chamar de bioindicação, em que cada sistema biológico - organismo, população, comunidade - tem a capacidade de responder, de maneira direta ou indireta, a efeitos sobre o meio, sejam eles naturais, antrópicos ou modificados antropicamente (BIANCHI et al., 2010). Em razão, principalmente, do seu ciclo de vida, da alta sensibilidade 
apresentada nas primeiras fases de desenvolvimento e da moderada tolerância à variabilidade ambiental (HOLT; MILLER, 2010), o uso de invertebrados aquáticos em testes de toxicidade fornece um importante suporte na determinação de impactos químicos no meio ambiente (GHERARDI-GOLDSTEIN et al., 1990).

Os microcrustáceos de água doce, como a Daphnia magna (Cladocera, Crustacea), que, na fase adulta, atinge de 5 a $6 \mathrm{~mm}$ de tamanho, têm sido amplamente utilizados como indicador biológico em estudos de controle da qualidade da água (LE et al., 2016) e em testes de toxicidade na avaliação de efluentes, porque eles têm alta sensibilidade, disponibilidade e abundân- cia, tem ciclo de vida curto (cerca de 60 dias, a partir desse ponto a taxa reprodutiva diminui e a mortalidade aumenta), alta fecundidade, reproduzem-se por partenogênese (reprodução assexuada, da qual originam-se apenas fêmeas) e apresentam facilidade de cultivo em laboratório. São ainda considerados como padrão internacional para testes de toxicidade aguda (KNIE; LOPES, 2004).

O presente estudo objetivou, em virtude da eficiência do microcrustáceo como indicador de qualidade ambiental, avaliar o grau de toxicidade aguda em dafinídeos, determinada pelo uso de três agrotóxicos: o glifosato, a atrazina e o imidacloprido.

\section{MATERIAIS E MÉTODOS}

Para avaliar os efeitos dos agrotóxicos, foram adotados microcrustáceos da espécie Daphnia magna como indicadores biológicos, por serem mais apropriados para o monitoramento da realidade local. Os organismos fo- ram mantidos em condições controladas - câmaras de germinação com luminosidade difusa e fotoperíodo de 18 horas de luz e 6 horas de escuro, sendo a temperatura de $20 \pm 2^{\circ} \mathrm{C}$, conforme a NBR 12713 da ABNT (2009).

\section{Cultivo dos organismos}

Os organismos foram cultivados em dois meios: o meio básico, contendo os sais essenciais, característicos da água natural - cálcio $\left(\mathrm{CaCl}_{2} \cdot 2 \mathrm{H}_{2} \mathrm{O}\right)$, magnésio $\left(\mathrm{MgSO}_{4} \cdot 7 \mathrm{H}_{2} \mathrm{O}\right)$, potássio $(\mathrm{KCl})$, sódio $\left(\mathrm{NaHCO}_{3}\right)-$, e o meio $\mathrm{M} 4$, contendo elementos-traço e vitaminas. Esses meios apresentam diferentes concentrações e tipos de nutrientes, proporcionando condições nutricionais adequadas, aumentando as chances de desenvolvimento dos organismos-teste (ELENDT; BIAS, 1990).

As águas de cultivo (utilizadas para manutenção das culturas dos dafinídeos) e de diluição (usadas no preparo das soluções-estoque e soluções-teste) foram preparadas a partir de soluções feitas previamente com $\mathrm{pH}$ entre 7,6 e 8,0 e dureza total entre $175 \mathrm{mg}$ e $225 \mathrm{mg}$ $\mathrm{CaCO}_{3} / \mathrm{L}$. Tais águas preparadas foram aeradas por um período de 12 a 24 horas - tempo suficiente para que o pH se estabilizasse e o oxigênio dissolvido atingisse saturação. As daphnias foram alimentadas com algas da espécie Scenedesmus subspicatus (ABNT, 2009).

\section{Estudo das alterações estruturais e comportamentais}

As alterações estruturais e comportamentais dos bioensaios foram obtidas por meio da observação em estereomicroscópio com câmera acoplada (da marca Allition) e a olho nu, respectivamente. Foram considerados imóveis: organismos aparentemente mortos, incapazes de nadar na coluna d'água até 15 segundos após leve agitação do recipiente, e os que ficaram flutuando na superfície, ainda que apresentando movimento (KNIE; LOPES, 2004).

\section{Testes de sensibilidade e definitivo}

Para realização dos testes, fêmeas adultas de $D$. magna foram separadas um dia antes da realização do teste
O percentual de imobilidade foi observado para cada concentração testada e, ao final, determinou-se a concentração efetiva $\left(\mathrm{CE}_{50}\right)$, que é baseada na mortalidade de $50 \%$ dos indivíduos expostos (GHERARDI-GOLDSTEIN et al., 1990). Empregou-se o software da Microsoft Office Excel (2013) para a realização do cálculo da $\mathrm{CE}_{50}$.

utilizando-se peneiras de aço inox. No dia, foram utilizados neonatos de 2 a 26 horas de vida (ABNT, 2009). 
Foram realizados dois tipos de testes: os testes de sensibilidade, com duração de 24 horas, a fim de verificar se o bioindicador apresentava ou não condições de serem empregados na realização do trabaIho, e os testes definitivos, de 48 horas, utilizando os agrotóxicos.

Para o teste de sensibilidade, foi utilizado o dicromato de potássio, que é a substância de referência estabelecida pela ISO 6341 (ISO, 2012). Essa norma indica valores limites de sensibilidade entre 0,6 e 1,7 mg. $\mathrm{L}^{-1}$ de $\mathrm{CE}_{50}$ em 24 horas. Foram realizadas quadruplicatas de seis concentrações diferentes $(0,3 ; 0,5 ; 0,75 ; 0,95$; 1,1 e $\left.1,35 \mathrm{mg} . \mathrm{L}^{-1}\right)$, obtidas a partir da solução padrão de concentração igual a $100 \mathrm{mg} \cdot \mathrm{L}^{-1}$. Para realização dos testes, utilizou-se para cada concentração dez neonatos de daphnias.

Nos testes definitivos, utilizaram-se os seguintes agrotóxicos: o herbicida de nome comercial Atanor $48^{\circledR}$, contendo o princípio ativo glifosato (glifosato a 48\%); o herbicida Herbogex $A 500^{\circledR}$, contendo a atrazina a $50 \%$ como princípio ativo e o inseticida de nome comercial Evidence 700 WG $^{\circledR}$, contendo o princípio ativo imidacloprido (imidacloprido a 70\%). Esses agrotóxicos foram escolhidos com base em um levantamento dos mais importantes comercializados na área estudada, levando em consideração o potencial de contaminação e a classe toxicológica (SOUSA; NAVAL, 2013).

Tais agrotóxicos foram adotados no estudo porque o glifosato apresenta alto potencial de transporte tanto dissolvido em água quanto associado ao sedimento, indicando potencial risco tanto para água subterrânea quanto superficial. A atrazina e o imidacloprido apresentam alto potencial de transporte em água e médio potencial em transporte em sedimento.

Foram realizados dez testes, as concentrações utilizadas foram obtidas por meio de testes prévios e aleatórios, até que fosse obtido um intervalo de concentrações (Tabela 1), desde as que não fossem capazes de provocar reações nos bioensaios até as que causassem morte total ou quase total dos indivíduos utilizados nos testes.

Os testes foram realizados em recipientes acrílicos de $200 \mathrm{~mL}$, com $20 \mathrm{~mL}$ da solução-teste em cada um deles. Com o auxílio de uma pipeta plástica, foram colocadas 10 daphnias em cada recipiente, os quais foram cobertos com papel-alumínio, a fim de evitar contaminação por agentes químicos presentes no ar e, em seguida, foram colocados em uma bandeja e encaminhados para a incubadora a $20^{\circ} \mathrm{C}$. Essas permaneceram no

\section{Tabela 1 - Concentrações dos agrotóxicos empregados nos testes definitivos para determinar a toxicidade em indicadores da qualidade da água (Daphnia magna).}

\begin{tabular}{|l|c|c|c|}
\hline \multirow{2}{*}{ Testes } & \multicolumn{3}{|c|}{ Concentrações $\left(\mathrm{mg}^{-1}\right)$} \\
\cline { 2 - 4 } & Glifosato & Atrazina & Imidacloprido \\
\hline 1 & 9,6 & 0,5 & 80 \\
\hline 2 & 16,8 & 1,5 & 110 \\
\hline 3 & 19,2 & 3,0 & 150 \\
\hline 4 & 21,6 & 5,5 & 190 \\
\hline 5 & 24,0 & 7,0 & 220 \\
\hline 6 & 26,4 & 8,5 & 250 \\
\hline 7 & 31,2 & 10 & 270 \\
\hline 8 & 33,6 & 11 & 300 \\
\hline 9 & 38,4 & 12,5 & 325 \\
\hline 10 & 48,0 & 15 & 350 \\
\hline
\end{tabular}


escuro e sem alimentação por um período de 48 horas (ABNT, 2009; KNIE; LOPES, 2004).

Os testes de sensibilidade utilizando o dicromato de potássio $\left(\mathrm{K}_{2} \mathrm{CrO}_{4}\right)$ obtiveram uma $\mathrm{CE}_{50}$ de $0,77 \mathrm{mg} \mathrm{L}^{-1}$ após exposição de 24 horas, mostrando que os organismos-teste utilizados se encontravam em condições adequadas para a execução dos ensaios definitivos com os agrotóxicos (Figura 1), uma vez que a ISO 6341 (ISO, 2012) indica valores limites de sensibilidade entre $0,6 \mathrm{mg} \mathrm{L}^{-1}$ e $1,7 \mathrm{mg} \cdot \mathrm{L}^{-1}$ de $\mathrm{CE}_{50}$ em 24 horas.

A determinação da classe de toxicidade dos agrotóxicos foi feita baseada na tabela proposta por Zucker (1985). Com base nela, foi possível saber em qual classe estavam adotando o valor da $\mathrm{CE}_{50}$ encontrado (Tabela 2).

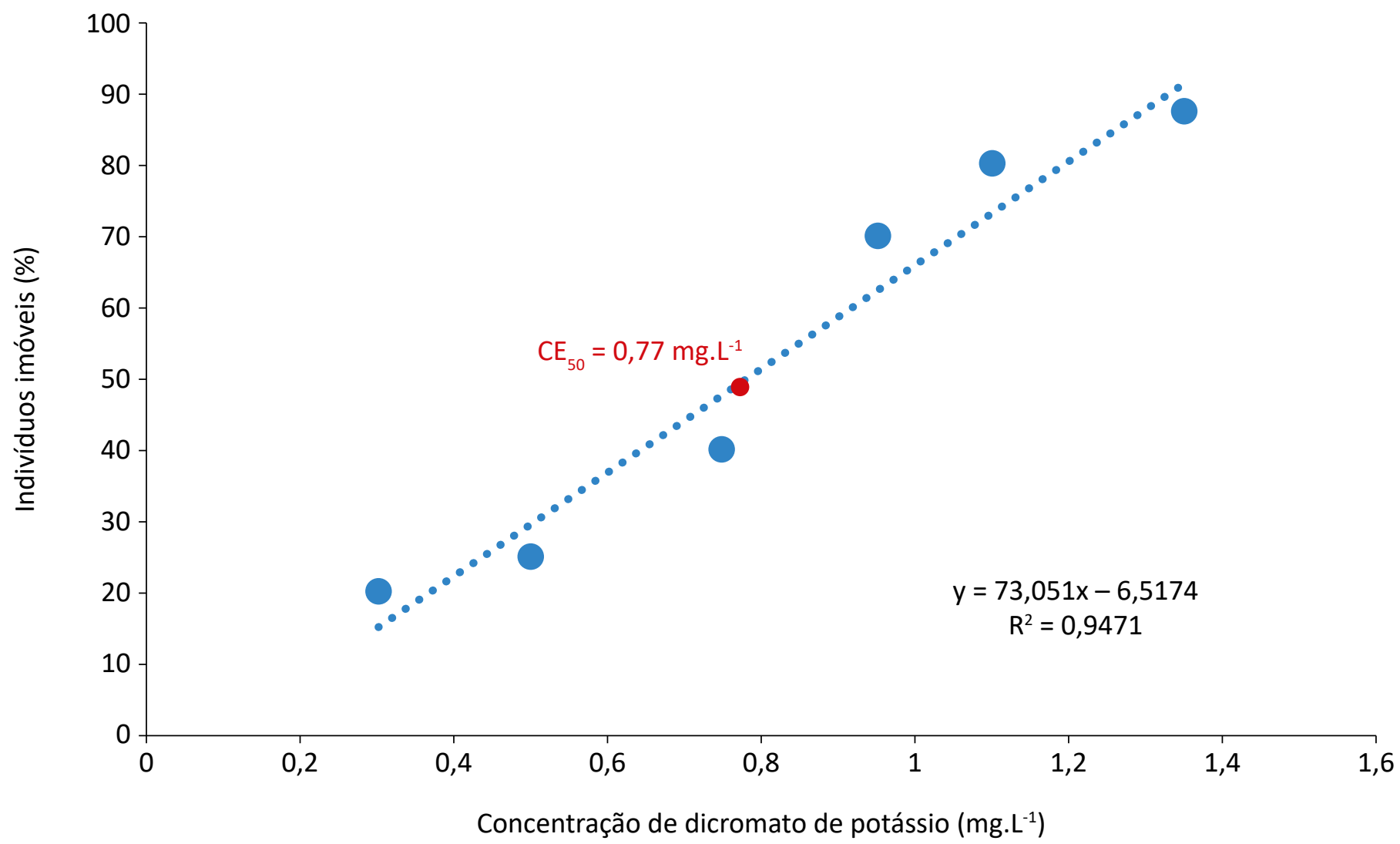

Figura 1 - Imobilidade da Daphnia magna, exposta a diferentes concentrações de dicromato de potássio, e (CE $\left.{ }_{50}\right)$.

Tabela 2 - Classe de toxicidade aguda de agrotóxicos para organismos aquáticos, segundo Zucker (1985).

\section{Classe de Toxicidade}

Extremamente tóxico

Altamente tóxico

Moderadamente tóxico

Ligeiramente tóxico

Praticamente não tóxico

\section{$\mathrm{CE}_{50}\left(\mathrm{mg} \mathrm{L}^{-1}\right)$}

$$
<0,1
$$

0,1 a 1,0

$$
>1,0 \text { e }<10
$$

$>10$ e $<100$

$>100$ 


\section{RESULTADOS E DISCUSSÃO}

Nos ensaios definitivos utilizando os agrotóxicos, primeiramente foram observadas alterações comportamentais e estruturais. Os que sobreviveram aos testes não apresentaram alterações na estrutura física e nem na forma de natação. Os imóveis permaneciam no fundo dos recipientes e paralisados. Estes apresentaram modificações na morfologia (Figura 2), com deformações nas carapaças (distorção do formato original, aparência espalhada causando impressão de aumento de tamanho) e também no tubo digestivo, que se encontrava interrompido (Figura 2). Fuzinatto (2009), utilizando o mesmo microcrustáceo, observou o encurtamento da espinha caudal como alteração na estrutura física.

Quanto às $\mathrm{CE}_{50}$ encontradas, notou-se que, com o aumento das concentrações dos agrotóxicos, se tinha proporcionalmente o aumento na imobilidade dos organismos. Os resultados para os valores de $\mathrm{CE}_{50}$ encontrados estão expostos na Figura 3.

Observa-se, na Figura $3 \mathrm{~A}$, que, para a concentração de 9,6 mg. $\mathrm{L}^{-1}$ do herbicida glifosato, não houve nenhum registro de morte. Para concentrações acima de $38,4 \mathrm{mg} . \mathrm{L}^{-1}$, tem-se um valor percentual alto de imobilidade das daphnias $(83,3 \%)$ e, para os organismos testados com a concentração $48,0 \mathrm{mg} \mathrm{L}^{-1}$, o efeito é deletério.

$\mathrm{A} \mathrm{CE}_{50}$ encontrada foi de $27,4 \mathrm{mg} \mathrm{L}^{-1}$. Pode-se dizer que esse é um herbicida ligeiramente tóxico, conforme a classificação proposta por Zucker (1985). Estudos anteriores sobre a toxicidade aguda do glifosato, para o mesmo microcrustáceo de água doce, encontraram valores de $\mathrm{CE}_{50}$ de $32 \mu \mathrm{g} \mathrm{L}^{-1}$ (BASTOS, 2013) e $\mathrm{CE}_{50}$ de $12 \mu \mathrm{g} \mathrm{L}^{-1}$ utilizando o herbicida glifosato Roundup ${ }^{\circledR}$, cuja formulação é mais tóxica (SARIGUL; BEKCAN, 2009), ou seja, valores abaixo do encontrado, podendo-se supor que o glifosato Atanor $48^{\circledR}$ apresenta formulação menos tóxica do que os utilizados pelos outros autores, necessitando assim de uma maior concentração até que provoque perturbação que acarrete a morte dos indivíduos-teste.

Para a concentração mínima testada de $0,5 \mathrm{mg} \mathrm{L}^{-1}$ (Figura 3B), observou-se que a concentração examinada provoca leve efeito nos organismos, enquanto a concentração máxima de $15 \mathrm{mg} \mathrm{L}^{-1}$ causa $96 \%$ de imobilidade, quase que a totalidade dos neonatos utilizados nos testes com o herbicida Herbogex $A 500^{\circledR}$, cujo prin-
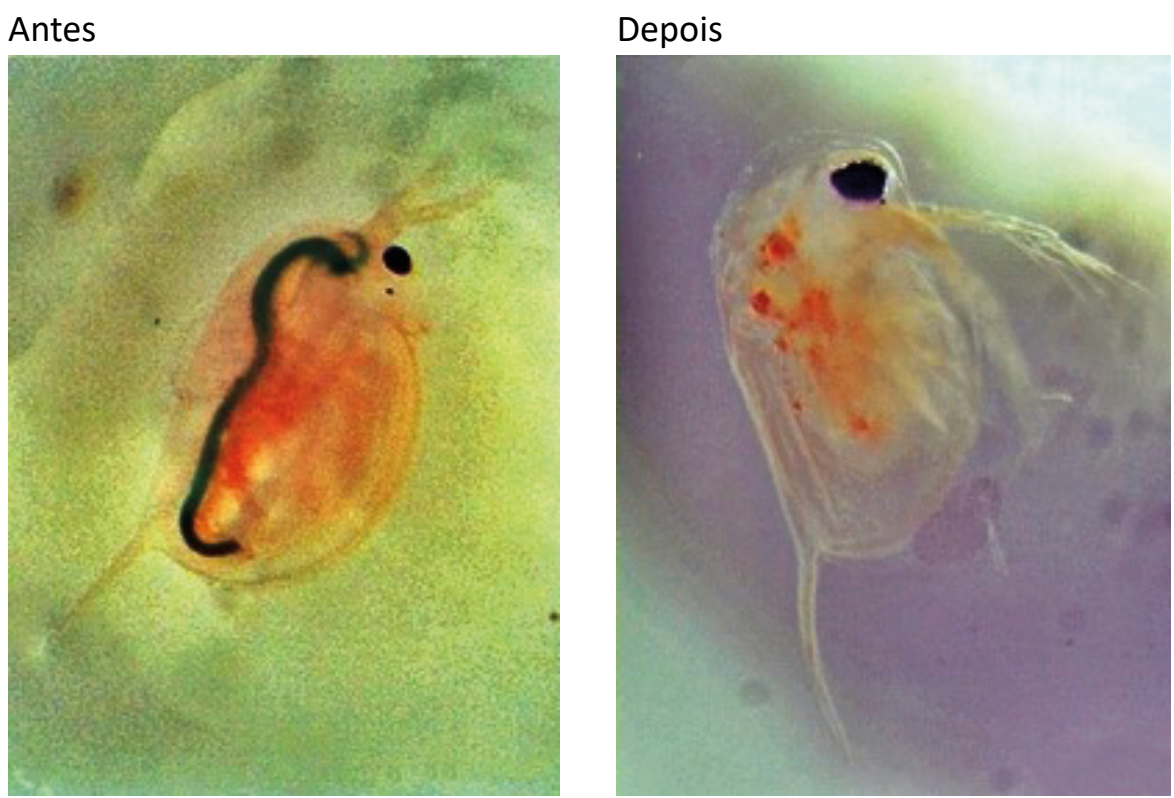

Figura 2 - Alterações estruturais em Daphnia magna com base em bioensaios que foram submetidos aos testes definitivos, em que se utilizou o herbicida contendo o princípio ativo glifosato, o herbicida atrazina e o inseticida imidacloprido. 

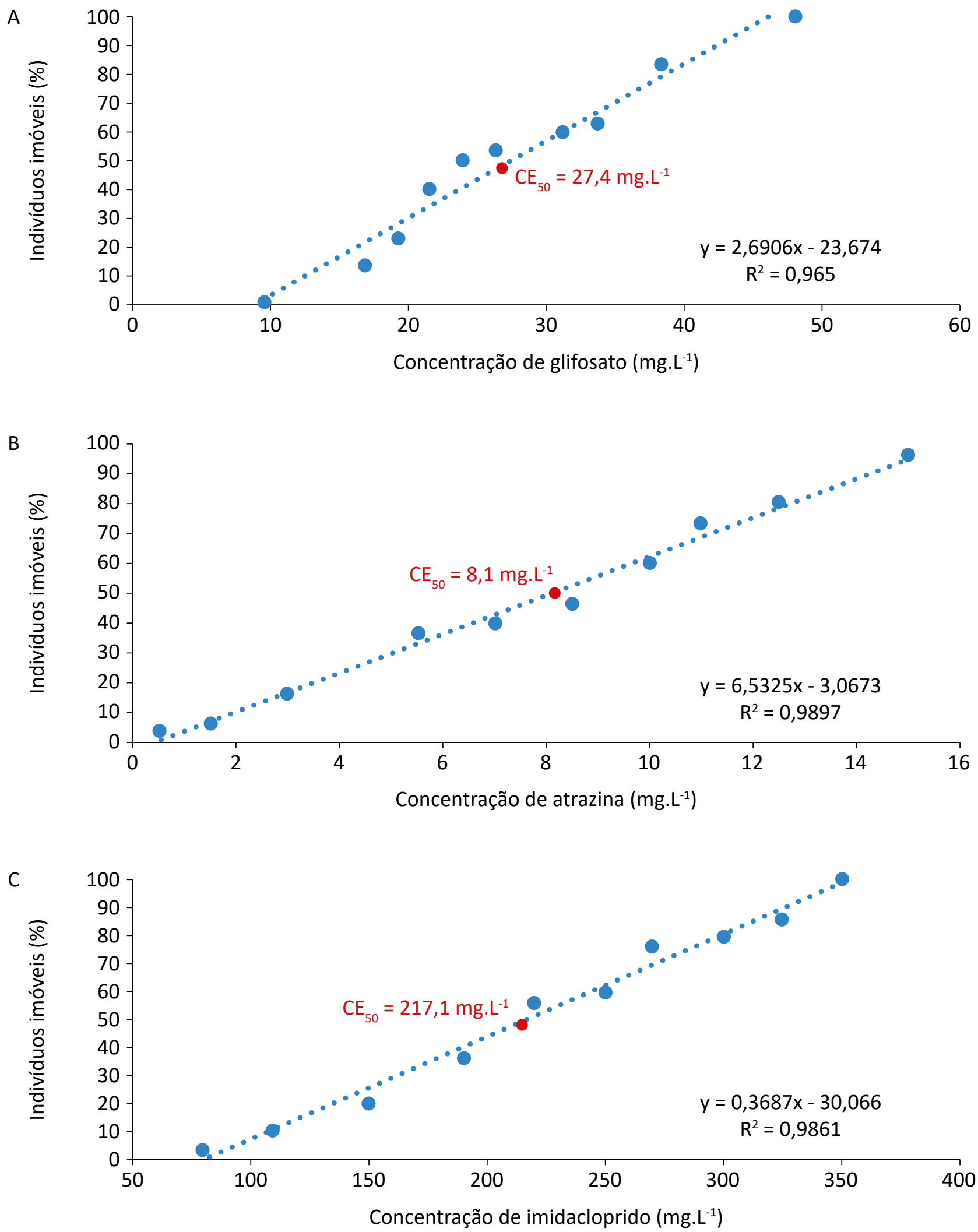

Figura 3 - Imobilidade da Daphnia magna, exposta a diferentes concentrações de glifosato (A), atrazina (B), imidacloprido (C); e concentração efetiva $\left(\mathrm{CE}_{50}\right)$. 
cípio ativo é a atrazina. $\mathrm{A} \mathrm{CE}_{50}$ obtida foi de $8,1 \mathrm{mg} \mathrm{L}^{-1}$, podendo-se afirmar que é moderadamente tóxico, conforme classificação de toxicidade aguda de agroquímicos para organismos aquáticos proposta por segundo Zucker (1985). Em estudos conduzidos por Moreira et al. (2014), foi obtido o valor de $50,4 \mathrm{mg} \mathrm{L}^{-1}$ para o mesmo princípio ativo, porém a marca comercial utilizada foi o herbicida atrazina Atanor $\mathrm{SC}^{\circledR}$. Utilizando o mesmo microcrustáceo, encontrou-se também na literatura uma $\mathrm{CE}_{50}$ para a atrazina de $26,9 \mathrm{mg} \mathrm{L}^{-1}$ (FREITAS; ROCHA, 2012).

Para o inseticida imidacloprido, a concentração de $80 \mathrm{mg} \mathrm{L}^{-1}$, mesmo sendo a mais baixa, apresenta efeito de mortalidade sobre as daphnias, enquanto a de $350 \mathrm{mg} \mathrm{L}^{-1}$ é causadora da mortandade de $100 \%$ da população exposta ao agente químico (Figura $3 \mathrm{C}$ ). Encontrou-se $\mathrm{CE}_{50}$ de $217,1 \mathrm{mg} \mathrm{L}^{-1}$, apontando ser praticamente não tóxico (ZUCKER, 1985).

De modo geral, para Daphnia magna, a concentração efetiva média $\left(\mathrm{CE}_{50}\right)$ - baseada na mortalidade de $50 \%$ dos indivíduos expostos - obtida neste estudo para os três agrotóxicos difere dos resultados encontrados em outros estudos (MOREIRA et al., 2014; BASTOS, 2013; IEROMINA et al., 2014; FREITAS; ROCHA, 2012; SARIGUL; BEKCAN, 2009). Isso corrobora o fato de que a utilização de agrotóxicos de marcas comerciais distintas proporciona diferentes resultados.

Especificamente no caso da atrazina (FOLT et al., 1999; MAYASICH et al., 1987), os efeitos tóxicos podem aumentar com o aumento da temperatura, sendo assim, as diferenças de $\mathrm{CE}_{50}$ devem ser tratadas com cuidado, pois podem sofrer variações. Outro fator que pode ser levado em consideração é a eventual utilização de indivíduos com sensibilidades diferentes, ou ainda a condições de cultura e de ensaios distintas no que diz respeito ao fotoperíodo, temperatura, dureza, tempo de exposição e a composição do meio de cultura.

O glifosato e o imidacloprido são toxicologicamente classificados como pouco e moderadamente tóxico (ANVISA, 2019), no entanto destaca-se que, tratando-se de efeitos ambientais, são perigosos e representam riscos (EVIDENCE, 2011). Apesar de o glifosato ser pouco tóxico, ele é utilizado em grande escala, o que se torna um agravante. O glifosato é o ingrediente ativo mais comercializado no Brasil
(REBELO et al., 2010). A propriedade física que se pode salientar é a sua miscibilidade. Esse agrotóxico é totalmente miscível em água, quando se encontra em condições ambientais de temperatura e pressão adequadas, e apresenta alto transporte tanto em água (altamente hidrossolúvel) quanto em sedimento. Essa capacidade de misturar-se na água permite-lhe ser apontado como potencial poluidor ambiental. Isso implica dizer que é necessária atenção e cuidado quanto à sua aplicação e uso excessivo, por apresentar potencial risco de contaminação às águas superficiais e subterrâneas.

A atrazina apresenta uma hidrólise ácida ou alcalina que produz um de seus mais abundantes produtos de degradação, a hidroxiatrazina (ESSER et al., 1985). É pouco miscível em água e a solubilidade se vê aumentada em soluções cujo pH é menor que 2,0 .

Quanto ao imidacloprido, sua solubilidade é considerada dispersível em água. No entanto, trata-se de um princípio ativo bastante comercializado (SOUSA; NAVAL, 2013). Os riscos são evidenciados em estudos realizados pela Agência de Proteção Ambiental (EPA, 1994), os quais mostram que o imidacloprido pode apresentar tempo de meia-vida em solos superior a um ano e elevado potencial de contaminação de águas superficiais e subsuperficiais.

Considerando os evidentes riscos à biota causados pela presença de agrotóxicos, os Estados Unidos e a Europa estabeleceram regulamentos (USEPA, 2007; EUROPEAN UNION, 2009) para o seu registro, exigindo que o destino ambiental e os dados de ecotoxicidade sejam fornecidos para os metabólitos. A avaliação do risco ecológico é necessária, usando os dados de destino e ecotoxicidade do ambiente para cada composto, tomando-se em conta as orientações de risco dos agrotóxicos para organismos aquáticos (EFSA, 2013; USEPA, 2004).

A avaliação do risco sobre um microrganismo aquático, por meio da presença de um metabólito, deve ser realizada quando o metabólito: contiver um toxicóforo, for formado rapidamente de um composto original no ambiente e/ou ser mais hidrofóbico que o composto original (IWAFUNE, 2018). A avaliação de risco para os metabólitos é recomendada quando a toxicidade para organismos aquáticos for semelhante ou superior à do composto original (IWAFUNE, 2018). 


\section{CONCLUSÕES}

Com base em bioensaios submetidos aos testes definitivos para o herbicida glifosato (Atanor $48^{\circledR}$ ), no que tange às $\mathrm{CE}_{50}$, à medida que a concentração desse agrotóxico foi aumentada, houve aumento na imobilidade dos organismos, evoluindo para o efeito deletério.

O glifosato (Atanor $48^{\circledR}$ ) foi categorizado como ligeiramente tóxico em função da $\mathrm{CE}_{50}$ encontrada, associando-se à formulação menos tóxica. Nos testes com o herbicida Herbogex $A 500^{\circledR}$, cujo princípio ativo é a atrazina, a $\mathrm{CE}_{50}$ indicou que esse princípio ativo é moderadamente tóxico. Para o inseticida imidacloprido, a concentração empregada, mesmo sendo baixa, apresenta efeito de mortalidade sobre as daphnias, causando a mortandade de $100 \%$ da população exposta ao agente químico. No entanto, encontrou-se $\mathrm{CE}_{50}$ indicando ser praticamente não tóxico, de acordo com a classificação proposta por Zucker (1985).

Para o glifosato, observou-se a miscibilidade, o que caracteriza esse princípio ativo como altamente hidrossolúvel, o que o torna um potencial poluidor para as águas superficiais e subterrâneas.

Quanto às alterações estruturais, como a deformação nas carapaças e a interrupção do tubo digestivo, elas foram observadas nos dafinídeos quando submetidos aos testes, o que pode afetar a diversidade da biota aquática.

\section{REFERÊNCIAS}

AGÊNCIA NACIONAL DE VIGILÂNCIA SANITÁRIA (ANVISA). Publicada reclassificação toxicológica de agrotóxicos. ANVISA, 2019. Disponível em: <http://portal.anvisa.gov.br/noticias/-/asset_publisher/FXrpx9qY7FbU/content/ publicada-reclassificacao-toxicologica-de-agrotoxicos-/219201?p_p_auth=DepDKOBJ\&inheritRedirect=false $>$. Acesso em: 3 set. 2019.

ARRAES, A.I.O.M.; LONGHIN, S.R. Otimização de ensaio de toxicidade utilizando o bioindicador Allium cepa como organismo teste. Enciclopédia Biosfera, Goiânia, v. 8, n. 14, p. 1959, 2012.

ASSOCIAÇÃO BRASILEIRA DE NORMAS TÉCNICAS (ABNT). NBR 12713: Ecotoxicologia Aquática - Toxicidade Aguda Método de ensaio com Daphnia spp. (Cladocera, Crustácea). Rio de Janeiro: ABNT, 2009.

BASTOS, D.N. Toxicidade do Herbicida Glifosato em Daphnia magna e pós-larvas de Rhamdia quelen. Dissertação (Mestrado) - Centro de Engenharias e Ciências Exatas, Universidade Estadual do Oeste do Paraná, Toledo, 2013.

BATTAGLIN, W.A.; MEYER, M.T.; KUIVILA, K.M.; DIETZE, J.E. Glyphosate and its degradation product AMPA occur frequently and widely in US soils, surface water, groundwater, and precipitation. Journal of the American Water Resources Association, v. 50, n. 2, p. 275-290, 2014. https://doi.org/10.1111/jawr.12159

BIANCHI, M.O.; CORREIA, M.E.F.; RESENDE, A.S.; CAMPELLO, E.F.C. Importância de estudos ecotoxicológicos com invertebrados do solo. Seropédica: Embrapa Agrobiologia, 2010.

CALIFORNIA DEPARTMENT PESTICIDES REGULATION (CDPR). Environmental Pathways of Imidacloprid. CDPR, 2016. Disponível em: <https://www.cdpr.ca.gov/docs/risk/rcd/imidacloprid.pdf>. Acesso em: set. 2019.

COUPE, R.H.; KALKHOFF, S.J.; CAPEL, P.D.; GREGOIRE, C. Fate and transport of glyphosate and aminomethylphosphonic acid in surface waters of agricultural basins. Pest Management Science, v. 68, n. 1, p. 16-30, 2012. https://doi. org/10.1002/ps.2212

DARUICH, J.; ZIRULNIK, F.; GIMENEZ, M.S. Effect of the herbicide glyphosate on enzymatic activity in pregnant rats and their fetuses. Environmental Research, v. 85, n. 3, p. 226-231, 2001. https://doi.org/10.1006/enrs.2000.4229 
DORES, E.F.G; DE-LAMONICA-FREIRE, E.M. Contaminação do ambiente aquático por pesticidas. Estudo de caso: Águas usadas para abastecimento humano em Primavera do Leste. Mato Grosso. Análise preliminar. Química Nova, v. 24, n. 1, p. 27-36, 2001. http://dx.doi.org/10.1590/\$0100-40422001000100007

ELENDT, B.P.; BIAS, W.R. Trace nutrient deficiency in Daphnia magna cultured in standard medium for toxicity testing: effects of the optimization of culture conditions on life history parameters of Daphnia magna. Water Research, v. 24, n. 9, p. 1157-1167, 1990. https://doi.org/10.1016/0043-1354(90)90180-E

ENVIRONMENTAL PROTECTION AGENCY (EPA). Imidacloprid Pesticide Factor Sheet. Washington, D.C.: EPA, 1994.

ESSER, H.O.; DUPUIS, G.; EBERT, E.; MARCO, G.; VOGEL, C. Triazines. In: KEARNEY, P.C.; KAUFMAN, D.D. (orgs.). Herbicides: Chemistry, degradation and mode of action. Nova York: Kaufmamn, 1985. v. 1. p. 129-208.

EUROPEAN UNION. Regulation (EC) No. 1107/2009 of the European parliament and of the council. Off. J. Eur. Union. European Union, 2009.

EUROPEAN FOOD SAFETY AUTHORITY (EFSA). Guidance on tiered risk assessment for plant protection products for aquatic organisms in edge-of-field surface waters. 3290. EFSA, 2013.

EVIDENCE 700 WG: agroquímico. Ficha de informações de Segurança de Produtos químicos. São Paulo: Bayer, 2011.

FERREIRA, M.L.P.C. A pulverização aérea de agrotóxicos no Brasil: cenário atual e desafios. Revista Direito Sanitário, v. 15, n. 3, p. 18-45, 2014. https://doi.org/10.11606/issn.2316-9044.v15i3p18-45

FOLT, C.L.; CHEN, C.Y.; MOORE, M.V.; BURNAFORD, J. Synergism and antagonism among multiple stressors. Limnology and Oceanography, v. 44, n. 3, parte 2, p. 864-877, 1999. https://doi.org/10.4319/lo.1999.44.3_part_2.0864

FREITAS, E.C.; ROCHA, O. Acute and chronic effects of atrazine and sodium dodecyl sulfate on the tropical freshwater cladoceran Pseudosida ramosa. Ecotoxicology, v. 21, n. 5, p. 1347-1357, 2012. https://doi.org/10.1007/s10646-0120888-1

FUZINATTO, C.F. Avaliação da Qualidade da Água de Rios Localizados na Ilha de Santa Catarina utilizando Parâmetros Toxicológicos e o Índice de Qualidade de Água. 243f. Dissertação (Mestrado) - Programa de Pós-Graduação em Engenharia Ambiental, Universidade Federal de Santa Catarina, Florianópolis, 2009.

GHERARDI-GOLDSTEIN, E. et al. Procedimentos para utilização de testes de toxicidade no controle de efluentes líquidos. São Paulo: CETESB, 1990.

GOULSON, D. Review: an over view of the environmental risks posed by neonicotinoid insecticides. Journal of Applied Ecology, v. 50, n. 4, p. 977-987, 2013. https://doi.org/10.1111/1365-2664.12111

HOLT, E.A.; MILLER, S.W. Bioindicators: using organisms to measure environmental impacts. Nature, v. 3, n. 10, p. 8-13, 2010.

IEROMINA, O.; PEIJNENBURG, W.J.G.M.; SNOO, G.; MÜLLER, J.; KNEPPER, T.P.; VIJVER, M.G. Impact of Imidacloprid on Daphnia magna under different food quality regimes. Environmental Toxicology Chemistry, v. 33, n. 3, p. 621-631, 2014. https://doi.org/10.1002/etc.2472

INTERNATIONAL AGENCY FOR RESEARCH ON CANCER (IARC). Evaluation of five organophosphate insecticides and herbicides. IARC, 2015. v. 12. Disponível em: <https://www.iarc.fr/news-events/iarc-monographs-volume-112evaluation-of-five-organophosphate-insecticides-and-herbicides/>. Acesso em: ago. 2019.

INTERNATIONAL STANDARD (ISO). ISO 6341. Water quality. Determination of the inhibition of the mobility of Daphnia magna Straus (Cladocera, Crustacea). Acute toxicity test. ISO, 2012. 
IWAFUNE, T. Studies on the behavior and ecotoxicity of pesticides and their transformation products in a river. Journal of Pesticides Science, v. 43, n. 4, p. 297-304, 2018. http://doi.org/10.1584/jpestics.J18-01

KHATRI, N.; TYAGI, K.S. Influences of natural and anthropogenic factors on surface and groundwater quality in rural and urban areas. Front Life Science, v. 8, n. 1, p. 23-39, 2015. https://doi.org/10.1080/21553769.2014.933716

KNIE, J.L.W.; LOPES, E.W.B. Testes ecotoxicológicos: métodos, técnicas e aplicações. Florianópolis: FATMA / GTZ, 2004.

KURWADKAR, S.T.; DEWINNE, D.; WHEAT, R.; MCGAHAN, D.G., MITCHELL, F.L. Time dependent sorption behavior of dinotefuran, imidacloprid and thiamethoxam. Journal of the Environmental Science Health, Part B, v. 48, n. 4, p. 237-242, 2013. https://doi.org/10.1080/03601234.2013.742412

LE, Q.A.V.; SEKHON, S.S.; LEE, L.; KO, H.J.; MIN, J. Daphnia in water quality biomonitoring - "omic" approaches. Toxicology Environmental Health Science, v. 8, n. 1, p. 1-6, 2016. https://doi.org/10.1007/s13530-016-0255-3

LIU, Z.; WANG, Y.; ZHU, Z.; YANG, E.; FENG, X.; FU, Z.; JIN, Y. Atrazine and its main metabolite salterthe locomotor activity of larval zebrafish (Daniorerio). Chemosphere, v. 148, p. 163-170, 2016. https://doi.org/10.1016/j. chemosphere.2016.01.007

MARC, J.; MULNER-LORILLON, O.; BOULBEN, S.; HUREAU, D.; DURAND, G.; BELLÉ, R. Pesticide Roundup provokes cell division dysfunction at the level of CDK1/cyclin B activation. Chemical Research Toxicology, v. 15, n. 3, p. 326-331, 2002. https://doi.org/10.1021/tx015543g

MAYASICH, J.M.; KARLANDER, E.P.; TERLIZZI JUNIOR, D.E. Growth responses of Nannochloris oculata Droop and Phaeodactylum tricornutum Bohlin to the herbicide atrazine as influenced by light intensity and temperature in unialgal and bialgal assemblage. Aquatic Toxicology, v. 10, n. 4, p. 187-197, 1987.

MERCURIO, P.; FLORES, F.; MUELLER, J.F.; CARTER, S.; NEGRI, A.P. Glyphosate persistence in seawater. Marine Pollution Bulletin, v. 85, n. 2, p. 385-390, 2014. https://doi.org/10.1016/j.marpolbul.2014.01.021

MOREIRA, R.A.; MANSANO, A.S.; SILVA, L.C.; ROCHA, O. A comparative study of the acute toxicity of the herbicide atrazine to cladocerans Daphnia magna, Ceriodaphnia silvestrii and Macrothrix flabelligera. Acta Limnologica Brasiliensia, v. 26, n. 1, p. 1-8, 2014. http://dx.doi.org/10.1590/S2179-975X2014000100002

OSTEN, J.R.; DZUL-CAAMAL, R. Glyphosate Residues in Groundwater, Drinking Water and Urine of Subsistence Farmers from Intensive Agriculture Localities: A Survey in Hopelchén, Campeche, Mexico. International Journal of Environmental Research Public Health, v. 14, n. 6, p. 595, 2017. https://doi.org/10.3390/ijerph14060595

PARMAR, T.K.; RAWTANI, D.; AGRAWAL, Y.K. Bioindicators: the natural indicator of environmental pollution. Frontiers in Life Science, v. 9, n. 2, p. 110-118, 2016. https://doi.org/10.1080/21553769.2016.1162753

PROSEN, H. Fate and determination of triazine herbicides in soil. In: HASANEEN, M.N.A.E. (org.). Herbicides: Properties, Synthesis and Control of Weeds. Rijeka: Intech, 2012. p. 43-58.

REBELO, R.M.; VASCONCELOS, R.A.; BUYS, B.D.M.C.; REZENDE, J.A.; MORAES, K.O.C.; OLIVEIRA, R.P. Produtos agrotóxicos e afins comercializados em 2009 no Brasil: uma abordagem ambiental, Instituto Brasileiro do Meio Ambiente e dos Recursos Naturais Renováveis. Brasília: IBAMA, 2010.

RIBEIRO, M.L.; LOURENCETTI, C.; PEREIRA, S.Y.; MARCHI, M.R.R. Contaminação de águas subterrâneas por pesticidas: avaliação preliminar. Química Nova, v. 30, n. 3, p. 688, 2007. http://dx.doi.org/10.1590/S0100-40422007000300031

RICHARD, S.; MOSLEMI, S.; SIPAHUTAR, H.; BENACHOUR, N.; SERALINI, G.E. Differential effects of glyphosate and roundup on human placental cells and aromatase. Environmental Health Perspective, v. 113, n. 6, p. 716-720, 2005. https://doi.org/10.1289/ehp.7728 
RONCO, A.E.; MARINO, D.J.G.; ABELANDO, M.; ALMADA, P.; APARTIN, C.D. Water quality of the main tributaries of the Paraná Basin: glyphosate and AMPA in surface water and bottom sediments. Environmental Monitoring and Assessment, v. 188, p. 458, 2016. https://doi.org/10.1007/s10661-016-5467-0

SARIGUL, Z.; BEKCAN, S. Acute toxicity of the herbicide glyphosate on Daphnia magna. Journal of Agricultural Science, v. 15, n. 2, p. 204-208, 2009.

SOUSA, E.S.; NAVAL, L.P. Análise de risco: Avaliação do potencial de contaminação de águas superficiais por agrotóxicos. In: SEMINÁRIO DE INICIAÇÃO CIENTÍFICA UFT, 2013, Palmas. Anais... Palmas.

THUYET, D.Q.; JORGENSON, B.C.; WISSEL-TYSON, C.; WATANABE, H.; YOUNG, T.M. Wash off of imidacloprid and fipronil from turf and concrete surfaces using simulated rainfall. Science Total Environment, v. 414, p. 515-524, 2012. https:// doi.org/10.1016/j.scitotenv.2011.10.051

TSYGANKOV, V.Y.; BOYAROVA, M.D.; LUKYANOVA, O.N.; KHRISTOFOROVA, N.K. Bioindicators of Organochlorine Pesticides in the Sea of Okhotsk and the Western Bering Sea. Archives of Environmental Contamination and Toxicology, v. 73, n. 2, p. 176-184, 2017. https://doi.org/10.1007/s00244-017-0380-2

UNITED STATES ENVIRONMENTAL PROTECTION AGENCY (USEPA). Basic Information about Pesticide Ingredients. USEPA, 2017. Disponível em: <https://www.epa.gov/ingredients-used-pesticide-products/basic-information-aboutpesticideingredients>. Acesso em: ago. 2018.

Hazards summary. USEPA, 2007. Disponível em: <https://pubchem.ncbi.nlm.nih.gov/ compound/2256\#section=Hazards-Summary>. Acesso em: set. 2019.

. Overview of the Ecological Risk Assessment Process in the Office of Pesticide Programs. Washington, D.C.: USEPA, 2004. Acesso em: set. 2019.

VAN STEMPVOORT, D.R.; SPOELSTRA, J.; SENGER, N.D.; BROWN, S.J.; POST, R.; STRUGER, J. Glyphosate residues in rural groundwater, Nottawasaga River watershed, Ontario, Canada. Pest Management Science, v. 72, n. 10, p. 1862-1872, 2016. https://doi.org/10.1002/ps.4218

ZUCKER, E. Hazard Evaluation Division: Standard Evaluation Procedure - Acute toxicity test for freshwater fish. Washington, D.C.: USEPA, 1985. 